\title{
Classical Fields and Quantum Time-Evolution in the
}

\section{Aharonov-Bohm Effect}

James Mattingly

February 14, 2007

\begin{abstract}
I display, by explicit construction, an account of the Aharonov-Bohm effect that employs only locally operative electrodynamical field strengths. The terms in the account are the components of the magnetic field of the solenoid at the location of electron, and even though the total field vanishes there, the components do not. That such a construction can be carried out demonstrates at least that whatever virtues they have for understanding and constructing new field theories, gauge fields in general make no metaphysical demands, and commit us to no novel ontology. I reflect on the significance of this for our understanding of quantum time evolution and conclude that we should think of quantized matter as interacting individually with the other matter in the systems of which it is a part.
\end{abstract}

Keywords: Aharonov-Bohm effect; electrodynamics; gauge theory; field theory. 


\section{Introduction}

This essay is a first attempt to try to understand more clearly the nature of linearity in some classical field theories, and how that linearity is manifested in the time-evolution of quantum theories. I will focus my attention, in this instance, on a narrow target, the Aharonov-Bohm (AB) effect. In what follows I will highlight a feature of electrodynamics involving quantum matter that has been neglected in analyses of the $\mathrm{AB}$ effect: net electromagnetic fields do not encode all of the features of the component electromagnetic fields in their interaction with quantum systems. The linearity of electrodynamics allows one to show that the $\mathrm{AB}$ effect arises purely as a result of the peculiar nature of quantum time-evolution, and that it is governed by component rather than net field quantities. The effect shows us nothing at all about the ontological status of the gauge field of electrodynamics.

Analyzing our physical theories using the techniques of gauge theory, and indeed using the insights gained therefrom to construct new physical theories, has resulted in significant theoretical advances in recent decades ${ }^{1}$. However these uses do not underwrite the various kinds of metaphysical adjustment claimed for them by the students of gauge theory. My aim here is to show that one can, if one wishes, deny any metaphysical novelty attendant to gauge theories in general, and toward this end I show that the Aharonov-Bohm (AB) effect poses no threat to locality, supervenience, or determinism - in short that it has nothing to teach us about metaphysics. Worries about the implications of the $\mathrm{AB}$ effect are largely responsible for the seeming plausibility of certain metaphysically novel, and otherwise unwelcome responses to gauge theory; we will be well rid of them. Here I demonstrate an entirely new construction of the Aharonov-Bohm effect, one that employs

\footnotetext{
${ }^{1}$ But see Martin (2002) for a critique.
} 
only electrodynamical field strengths (as opposed to potentials) and yet is completely local. There is no gauge dependence, nor do any non-local terms or integrals appear in the formulation. My argument is simple: since the one gauge theory we understand best is shown to require no metaphysical novelty, then general facts about gauge theories will have no interesting metaphysical implications. That is, since no new metaphysics is associated with a paradigm gauge theory, then basic facts about gauge theories as a class cannot require novel metaphysics. My aim is to establish the antecedent of this implication. A full discussion of how to extend this analysis to other gauge theories will be reserved for another occasion.

The construction is quite trivial: Calculate the magnetic (electromagnetic) field of a single element of the electric current (electromagnetic charge-current density). Calculate the infinitesimal effect of this field on the phase of the electron. Sum these results over all current elements. This procedure gives the phase change along the electron path for an infinitesimal element of path length. Now integrate the phase change, $\Delta \phi$ along the whole path for each separate component of the electron wave function and (in general) the electron wave functions will have undergone different phase changes and so the diffraction pattern will have shifted. However at no time will the electron have been in a region with a non-zero magnetic field. Thus distributivity fails for this interaction; that is, the electron's phase is sensitive to component fields not net field quantities. The interaction will have a form much like the cross-product of two vectors, and in the same way that distributivity fails for the cross-product, it fails for this interaction. I.e., for $\mathbf{B}_{i}$ the magnetic field of an element of current, there exists an $f$ such that $\sum_{i} f\left(\mathbf{B}_{i}\right)=\Delta \phi$; whereas there is no $g$ such that $g\left(\sum_{i} \mathbf{B}_{i}\right)=\Delta \phi$. The sum of the effects cannot be rewritten as the effect of a sum.

In what follows I will clarify the details of the construction, demonstrate that the construction 
works as advertised, and attempt to motivate the construction on physical grounds.

In Section 1 I present the essential features of the $\mathrm{AB}$ effect in its semiclassical form, leaving aside for now the question of how these features change when we consider quantum field theories. In Sections 2 and 3 I show how to understand the effect as arising from field quantities alone, and motivate my interpretation on physical grounds. In Section 4 I address the question of the "reality" of the quantum phase. I will conclude with some speculative suggestions for how we should begin addressing the question of the relation between gauge ontology and the enforcers of constraints in foundational physics.

\section{The AB effect}

Here I display the physical set-up leading to the AB effect and consider Aharonov and Bohm's original analysis. Even though the effect has been extensively studied in both the philosophical and physics literature, it will be well to revisit the key physical features of the experiment. Doing so will illuminate the construction to follow.

In the standard set-up for an AB-type experiment, we have the following items: an electron beam; a practically infinite or a toroidal solenoid ${ }^{2}$; a beam splitter; a detector screen. The beam is split into two components that take different paths around the solenoid and create a familiar interference pattern on the screen. When current flows through the solenoid the diffraction pattern at the screen is shifted from that observed when there is no current. The amount of the shift is determined by the quantity $\oint \mathbf{A} \cdot d \boldsymbol{l}$ where $\mathbf{A}$ is the vector potential generated by the current in the solenoid ${ }^{3}$.

\footnotetext{
${ }^{2}$ I concentrate on the practically infinite solenoid for clarity and ease of exposition, but what works for one works for the other as can be shown by, e.g., the method of images.

${ }^{3}$ In what follows I set $\hbar=1$.
} 
Consider Figure 1. On the left is the interference pattern with no current in the solenoid directly behind the screen, and on the right is the pattern when the current is switched on.

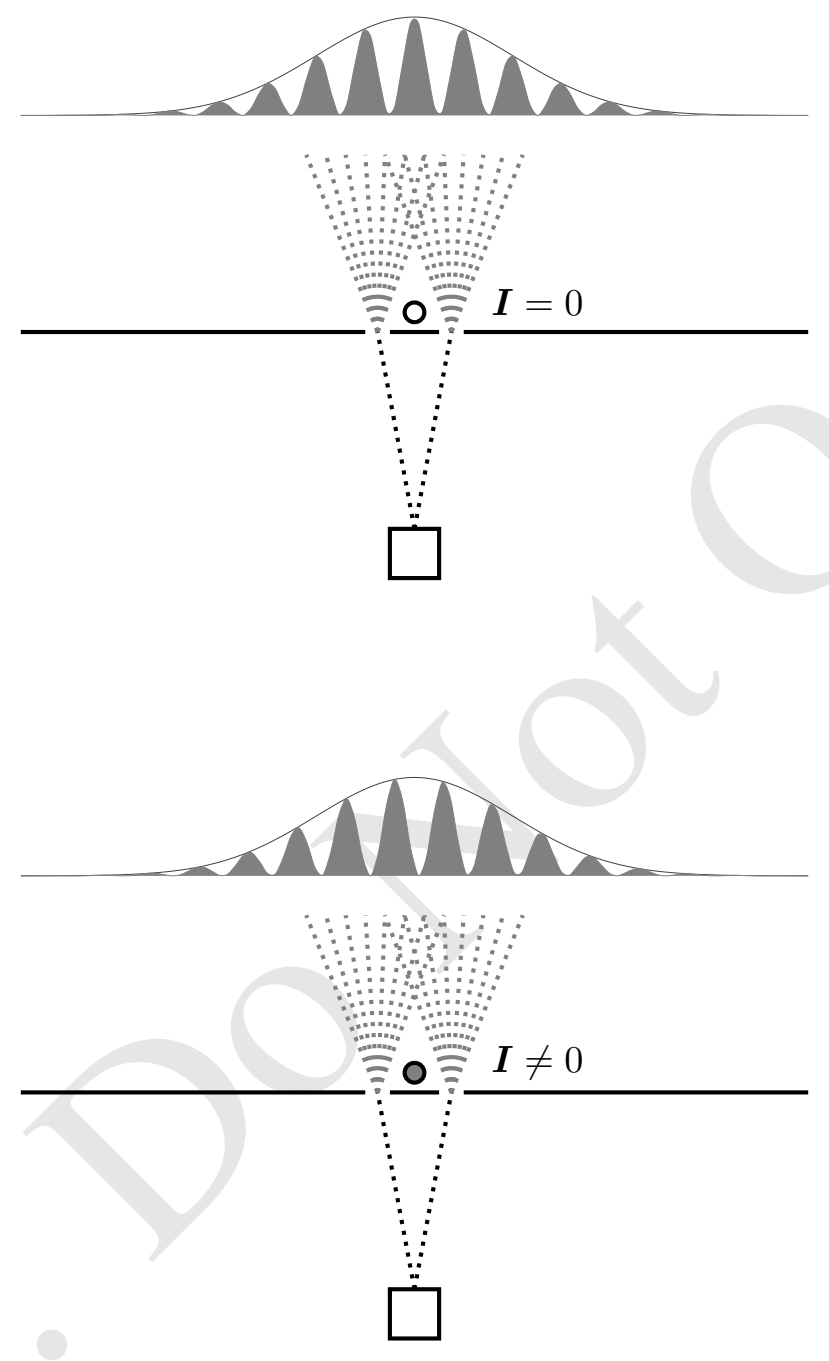

The way this effect is supposed to come about is via the change in the Lagrangian due to the presence of the solenoid. In quantum mechanics, the interaction Lagrangian for electrodynamics is given by $-e \phi+\frac{e}{c} \boldsymbol{v} \cdot \mathbf{A}$. As Aharonov and Bohm (1959) outline in their seminal paper about the effect, when we pass a coherent beam of electrons around a solenoid with magnetic field $\mathbf{B}=B \hat{\mathbf{z}}$ 
and vector-potential $\mathbf{A}=\frac{1}{\rho} B \hat{\boldsymbol{\phi}}$, we may consider the wave-function as split into two parts: $\psi_{1}$ and $\psi_{2}$. The phase of each beam will change along its path by a factor $\frac{e}{c} \int_{x_{i}}^{x_{f}} \mathbf{A} \cdot d \mathbf{x}$ and thus when the beams are brought back together the peaks of the interference pattern will have changed from the situation when there is no current flowing in the solenoid. According to Aharonov and Bohm, their thought experiment (now confirmed many times) shows that in quantum versions of electrodynamics "we are led to regard $A_{\mu}(x)$ as a physical variable." (491) Such a conclusion is especially striking because in the classical theory, the potentials are no more than calculational devices. Aharonov and Bohm make two related claims: that the potentials are ineleminable in the formulation of quantum mechanics; that their effect shows that the potentials are necessarily physically efficacious.

In the time since their paper was written it has been shown quite clearly that both claims are false as stated. See, e.g., DeWitt (1962), Belinfante (1962), Mattingly (2006). See as well Aharonov and Bohm (1962) in which they concede the formal correctness of DeWitt's and Belinfante's constructions. This much at least is plain: there are several different ways to formulate electrodynamics without potentials and still derive the $\mathrm{AB}$ effect within the theory. Naturally then it cannot be necessary to take the potentials to be physically efficacious. However, it has become dogma that we must either accept the reality of potentials in quantum mechanics and the problems attendant thereto or accept other even less palatable and/or even less intuitive conclusions (e.g., nonlocal field effects, or non-supervenience of the properties of a region on facts about that region alone, etc.). This latter dogma is somewhat more plausible than Aharonov and Bohm's 1959 claim, and one flavor of it is given in their 1962 paper. The fact remains that the vast majority of physicists and philosophers now do take the potentials as physically efficacious and then attempt to 
make clear what philosophical consequences flow from such an adoption. As far as I know I am the only philosopher who has attempted to show that the above dichotomy between non-locality and novel metaphysics is incomplete. In my (2006) I display a gauge independent "potential" (a proper, well-defined vector field) that can account for the effect, and I show how adopting this potential commits us to none of the thorny problems associated with other methods of rejecting gauge dependent potentials. But apparently even that construction has certain metaphysical novelties, principally that my field appears to carry information but neither momentum nor energy. Whether that appearance is correct will be considered another time, but for now I will set aside that interpretation and give what is, in any case, a much more straightforward option ${ }^{4}$.

The various interpretations that have been proposed to account for the AB effect are now wellknown. See, e.g., Belot (1998), Leeds (1999), Mattingly (2006), and Healey (1997), (1999), and also see especially Healey (forthcoming) for an exhaustive breakdown of all the various options and his reactions to them. I will not canvas those options here but will merely note that it is uncontroversially the consensus view that our understanding of electrodynamics must now change markedly - either by introducing some form of non-locality, or indeterminism, or by reifying the potential. I reject that view and claim instead that the $\mathrm{AB}$ effect shows us that field interactions play a different role in quantum theory than in classical. Of course that is well-known, but I argue that a full understanding of the $\mathrm{AB}$ effect highlights one important way in which those interactions conflict with our classical intuitions: quantum objects are affected not by the net field of the various objects with which they interact, but by the individual fields of those objects. Distributivity, in the sense outlined above fails for quantum interactions.

\footnotetext{
${ }^{4}$ Even if my potential does bear information, that feature of the potential is present in all analyses of the effect that do not make use of the field strengths alone. So we might think that even if some metaphysical novelty is present it is minimal in some sense.
} 


\section{The electromagnetic field}

In the long and fascinating early history of electrodynamics, from the observations of the peculiar effects of rubbed amber among the pre-socratics and of the stone of Heraclea (or magnet) described by Socrates in Plato's Ion, to the parlor tricks of the $17^{\text {th }}$ and $18^{\text {th }}$ Centuries, to the two-fluid model developed by Franklin, Faraday's contributions stand out for their striking clarity and for their impressive novelty and explanatory power. The field concept changed forever our understanding not only of electrodynamics, but of basic physics itself. Faraday's conception of substantial lines of electric and magnetic flux made possible our present understanding of forces between separated bodies mediated by locally acting fields. However, after Maxwell's mathematization of the theory, and his attempts to find mechanical models, and then Einstein's demonstration that electric and magnetic fields cannot be uniquely separated out of a more general "electromagnetic" field, Faraday's intuitions seem to have lost their hold on our imaginations.

I suggest that Faraday's insights can be adapted to the case of semiclassical electrodynamics (and possibly quantum electrodynamics) to give an intuitive picture of how to account for the $\mathrm{AB}$ effect completely locally in terms of electromagnetic field strengths alone. Thinking about the lines of flux encountered by the moving electron will make sense of the electromagnetic term in the Lagrangian. Notice now one key factor of Faraday's lines of flux: they do not vanish when in contact with each other, but rather "squeeze in" together. Beyond the imagery, what is the point of this suggestion? I claim that focusing on the individual components of the electromagnetic field-rather than the net field - will show quite clearly how the solenoid can appear to act where it is not. I claim further that an appeal to the components of the field will show that all that is involved in the AB effect are electromagnetic fields, and the peculiar non-distributive nature of quantum mechanical time 
evolution.

\subsection{Piecewise field descriptions}

The theory of electrodynamics is linear, and we can think about this in two ways. First it is linear in its solutions: for $\phi_{1}$ and $\phi_{2}$ possible solutions to the field equations, $a_{1} \phi_{1}+a_{2} \phi_{2}$ is also possible a solution for $a_{1}, a_{2} \in \mathbb{R}$. The values of $a_{1}, a_{2}$ are determined by boundary conditions.

Likewise the theory is linear in its solutions with respect to sources. That is, if the field of source $S_{1}$ is $\phi_{1}$ and that of $S_{2}$ is $\phi_{2}$ then the field of the combined sources $S_{1}, S_{2}$ is $\phi_{1}+\phi_{2}$. For example, the electric field of a point charge $q$ at a distance $r$ from the charge is $\mathbf{E}_{q}(r)=-\nabla \phi_{q}(r)=-\nabla \frac{q}{r}=\frac{q}{r^{2}} \hat{\mathbf{r}}$; for two charges $q_{1}, q_{2}$ the field at the origin with $q_{1}$ at $r_{1}$ and $q_{2}$ at $r_{2}$ is $\mathbf{E}_{q_{1}, q_{2}}(r)=-\nabla \phi_{q_{1}, q_{2}}(r)=$ $-\nabla\left[\frac{q_{1}}{r_{1}}+\frac{q_{2}}{r_{2}}\right]=\frac{q_{1}}{r_{1}^{2}} \hat{\mathbf{r}}_{1}+\frac{q_{2}}{r_{2}^{2}} \hat{\mathbf{r}}_{2}=\mathbf{E}_{q_{1}}+\mathbf{E}_{q_{2}}$.

I will take advantage of this second sense of linearity in what follows to characterize the change in the electron's phase as arising from field strengths alone. The solenoid itself is a spiral of current wrapped so tightly that it is essentially a stack of current loops. Likewise, these current loops are themselves arrays of moving charges confined to the loops. Thus, we build the full solenoid out of very many moving charges. Here's how.

\subsubsection{The field of a single element of current.}

For what follows see, e.g., (Jackson, 1975, ch 14). The Liénard-Wiechert potential at spacetime point $x$ of a single moving charge $q$ moving with 4 -velocity $v^{\mu}$ at $x^{\prime}$ is given by: $A^{\mu}(x)=$ $\frac{4 \pi}{c} \int d^{4} x^{\prime} D_{r}\left(x-x^{\prime}\right) J^{\mu}\left(x^{\prime}\right) . J^{\mu}(x)=q c \int d \tau v^{\mu}(\tau) \delta^{(4)}[x-r(\tau)]$ is the effective current of the charge. 
$D_{r}\left(x-x^{\prime}\right)=\frac{1}{2 \pi} \theta\left(x_{0}-x_{0}^{\prime}\right) \delta\left[\left(x-x^{\prime}\right)^{2}\right]$ is the retarded Green's function restricting the influence of the charge to the past light-cone of the observation point. The resulting expression for the magnetic field $\mathbf{B}$, in a frame where the velocity of the charge is $\boldsymbol{v}$, divides into a part that goes like $\frac{1}{R^{2}}$ and a part that goes like $\frac{1}{R}$ (the radiative term):

$$
\mathbf{B}(\mathbf{x})=q\left[\frac{\boldsymbol{\beta} \times \mathbf{n}}{\gamma^{2}(1-\boldsymbol{\beta} \cdot \mathbf{n})^{3} R^{2}}\right]_{r e t}+\frac{q}{c}\left[\frac{\mathbf{n} \times(\mathbf{n}-\boldsymbol{\beta}) \times \dot{\boldsymbol{\beta}}}{(1-\boldsymbol{\beta} \cdot \mathbf{n})^{3} R}\right]_{\text {ret }}
$$

Here $R$ is the spatial distance from the observation point to the location of the charge when it intersects the past light-cone of $\mathbf{x}, \boldsymbol{\beta}=\frac{\boldsymbol{v}}{c}, \gamma=\frac{1}{\sqrt{1-\frac{v^{2}}{c^{2}}}}, \dot{\boldsymbol{\beta}}=\frac{d \boldsymbol{\beta}}{d t}$, and $\mathbf{n}$ is a unit vector in that (spatial) direction.

The expression simplifies if $\dot{\boldsymbol{\beta}} \approx \mathbf{0}$, for then one need not consider the issue of radiation. We will consider only that case ${ }^{5}$, but nothing essential in the argument below turns on this choice. Now we have the magnetic field of a "steady-state" moving charge:

$$
\mathbf{B}(\mathbf{x})=q\left[\frac{\boldsymbol{\beta} \times \mathbf{n}}{\gamma^{2}(1-\boldsymbol{\beta} \cdot \mathbf{n})^{3} R^{2}}\right]_{r e t}
$$

So we see that the non-radiative component of the magnetic field is, to first order in $v / c$,

$$
\mathbf{B}(\mathbf{x})=\frac{q}{R_{r e t}^{2}} \frac{v}{c} \hat{\boldsymbol{v}} \times \mathbf{n}
$$

\footnotetext{
${ }^{5}$ The radiative case is of little interest here in any event since the influence of radiation may be characterized entirely in terms of its $\mathbf{B}$ and $\mathbf{E}$ fields, as is well known. Moreover, in all analyses of the effect the magnetic and electric fields are taken to vanish outside the solenoid, and that implies that the radiative term vanishes. It turns out however that for very many particles with very small charge such that $q N$ is constant and the current is steady state, the radiation term nearly vanishes independently of the state of motion of the particles. See Jackson (170 fn., and 697).
} 
and we recover the familiar $\frac{1}{R^{2}}$ dependence modified by a factor $\frac{v}{c}$.

\subsection{The field of a single loop of current.}

For further illustration we now confine the charge to a conducting loop and consider the magnetic field that results from adding more individual charges. For slow-moving charges in a relatively large loop, the acceleration of the individual charges is negligible, and we can continue to ignore the radiative component of the field. For an observation point in the plane of the loop, the direction of the field is either down into or up out of the plane of the loop, for the perpendicular component of the motion of the charge to the right or left of the observation point respectively. Now, because of the linearity of the theory, we can simply sum the first term on the right-hand side of Equation 2 over each of the charges in the loop:

$$
\mathbf{B}(\mathbf{x})=\sum_{i=1}^{n} q_{i}\left[\frac{\boldsymbol{\beta} \times \mathbf{n}}{\gamma^{2}(1-\boldsymbol{\beta} \cdot \mathbf{n})^{3} R^{2}}\right]_{r e t}
$$

\subsection{The field of an infinite solenoid.}

We could now continue and take a vertical array of current loops, tightly stacked, and extending upward and downward effectively infinitely, i.e., an infinite solenoid. We will not carry out this construction explicitly. First it would generate little insight because the mathematical burden is so great, and would be carried out in terms of complete sets of orthogonal functions rather than by way of elementary integrations over the field of the current loops themselves. This is because the field of each loop outside the plane of that loop is extraordinarily complicated. Second, the 
necessary illustration should already be clear: the net magnetic field arises by a vector sum of the magnetic fields of all the component elements of current (or the integration of them if one takes the current to be continuous). The magnetic field of the array of current loops is often said to be "confined" to the interior of the solenoid. However, as my brief discussion of Faraday's conception of flux was supposed to make plausible, one shouldn't think of this as a literal confinement. No bit of current is producing a distorted magnetic field, nor is any loop of current. Instead, the peculiar geometry of the set-up results in the net field of the device vanishing in the exterior of the solenoid. But the field still arises as the sum of very many undistorted magnetic fields. With sufficient time, one could carry out the series of sums

$$
\left.\mathbf{B}(\mathbf{x})=\sum_{\text {loops charges }} \sum_{\gamma^{2}(1-\boldsymbol{\beta} \cdot \mathbf{n})^{3} R^{2}}\right]_{r e t}+\frac{q}{c}\left[\frac{\mathbf{n} \times(\mathbf{n}-\boldsymbol{\beta}) \times \dot{\boldsymbol{\beta}}}{(1-\boldsymbol{\beta} \cdot \mathbf{n})^{3} R}\right]_{\text {ret }}
$$

or in our approximation

$$
\mathbf{B}(\mathbf{x})=\sum_{\text {loops charges }}\left[\frac{q v}{c R^{2}} \hat{\boldsymbol{v}} \times \mathbf{n}\right]_{\text {ret }}
$$

to find the complete field of the solenoid. The field is linear and arises from the vector sum of the fields of the individual current elements.

Just to be clear: each element of current generates a particular magnetic field, B, at a point $r$, and it turns out that when the fields of all current elements in a solenoid are added together, that sum vanishes outside the solenoid while inside the solenoid it need not. Consider Figure 2.3. The field of the single charge is circular. The many charges in the current loop all have circular fields but they all reinforce each other in the center of the loop while some oppose each other outside the loop. Finally the fields of the current loops reinforce each other in the interior of the solenoid and 
oppose each other in its exterior.
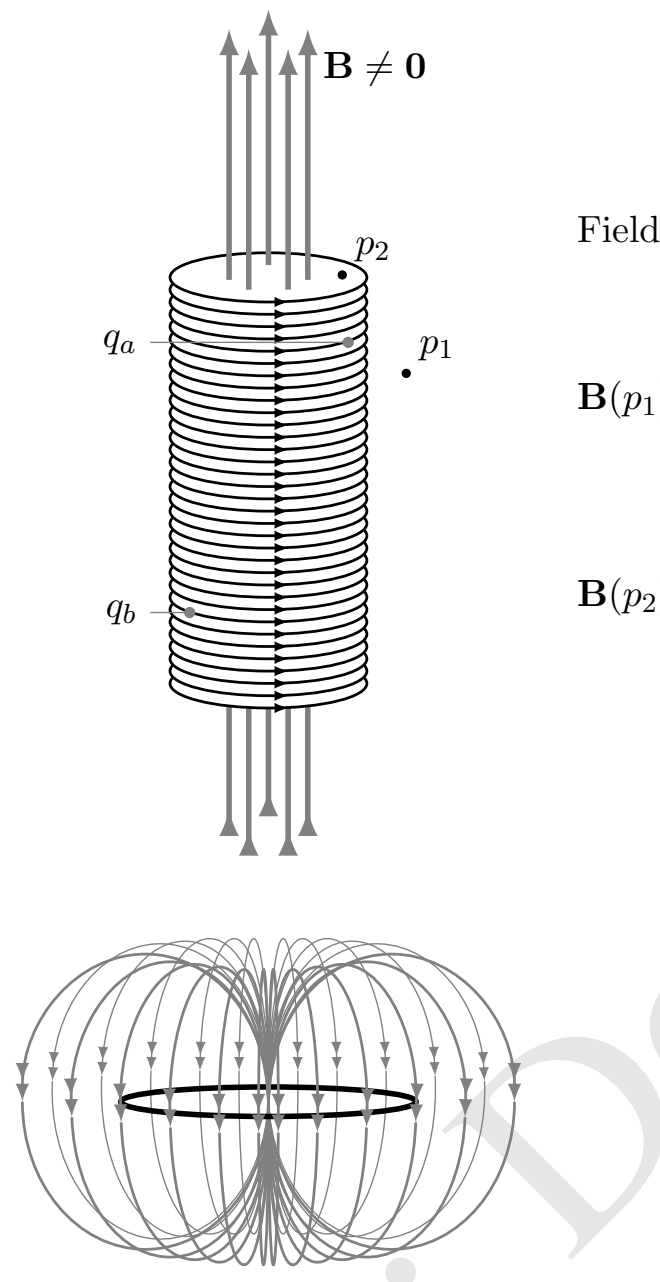

Field of a current loop.

$$
\mathbf{B}(R)=\sum_{i=1}^{n} q_{i}\left[\frac{\mathbf{n} \times \boldsymbol{\beta}}{\gamma^{2}(1-\boldsymbol{\beta} \cdot \mathbf{n})^{3}\left(R_{q_{i}}-R\right)^{2}}\right]_{\text {ret }}
$$

Field of a current element.

$$
\mathbf{B}(R)=\left[\frac{q}{R^{2}} \frac{v}{c} \mathbf{n} \times \hat{\boldsymbol{v}}\right]_{r e t}
$$

Figure 2.3. Components of the Solenoid. 
Now that we have been reminded of how the magnetic field comes about from the action of individual charges, we can easily see how to understand the phase shift of electrons moving in the neighborhood of a solenoid as arising directly from the magnetic fields of the current elements in the solenoid. It will, I hope, become obvious that one can tell a story that involves nothing more than field strengths and the motions of individual current elements. Neither novel fields nor indefinite potentials are required.

I now move on to the main part of the construction. We will see how the action of the magnetic fields of these current elements on the electron in the $\mathrm{AB}$ effect suffices to generate the phase shifts responsible for the effect.

\section{The AB Effect from magnetic fields alone}

In outline, the construction is as follows: calculate the phase change of the electron due to the field of a single element of current; sum over the current elements in a single loop; sum over loops in the solenoid. We will see that calculating the phase change due to each element of current before we sum over current elements can reproduce the total phase change required for the AB effect.

The force on a charge, $q$, moving in a magnetic field is $\frac{q}{c} \boldsymbol{v} \times \mathbf{B}$. But what is the potential energy associated with this force? Normally, for forces that are derivable from a potential, the difference in potential energy between two points is simply the integral from one point to the other of $\mathbf{F} \cdot d \mathbf{x}$. But magnetic forces are funny in that they do no work (since the force is always perpendicular to the velocity), and so for the actual travel of the particle, $\mathbf{F} \cdot d \mathbf{x}=\mathbf{F} \cdot d \boldsymbol{v} d t=0$. So in the case of magnetic interactions there is a question of what choice to make for the magnetic term that is 
analogous to the electrostatic potential energy term. Jackson (1975) offers the following (I think fairly typical) rationale: Since the interaction Lagrangian must be invariant under translations and Lorentz transformations, the coordinates cannot appear explicitly. And this then constrains the Lagrangian to the form (Jackson, 1975, p574):

$$
L_{i n t}=-\frac{e}{\gamma c} v_{\alpha} A^{\alpha} \quad \text { or } \quad L_{i n t}=-e \phi+\frac{e}{c} \boldsymbol{v} \cdot \mathbf{A}
$$

The following considerations may give some further physical insight into this choice. Given the parity between electric and magnetic fields that is made clear in the theory of relativity, presumably there should be some parity of reasoning in how we arrive at the potential energy associated with the two types of field. It cannot be, for example, that a shift to a frame where there is no magnetic field changes the work required to produce a given configuration. And yet there seems to be little discussion in the literature of how terms in $\boldsymbol{v} \cdot \mathbf{A}$ come to be associated with the potential energy term in the Lagrangian. The geometry of spacetime itself helps us to understand the requirement that the Lagrangian be covariant. But it does not give physical insight into the interactions recorded by that Lagrangian. What we do know is that something is doing the work to bring about and maintain the configuration of the system. So how should we think about this?

Here is Faraday's intuitive picture of the energy state between two moving charges. Between the charges, if they're moving co-linearly, is an array of oppositely directed lines of flux (the circular magnetic field created by moving charges), but these lines of flux are in the same direction for points to the outside of both charges. By coming closer together, the width of the array, and hence the total number of oppositely directed flux lines, is reduced. Similarly, for anti-co-linearly moving 
charges, the widening of the array decreases the number of oppositely directed flux lines - but here it is the opposing flux lines on the exterior of the array between the charges that decrease. As Faraday observed, magnets distribute themselves so as to increase the co-linear and decrease the anti-co-linear lines of flux between them. Packing anti-co-linear lines of flux together requires that work be done on the field. One needn't adopt this interpretation of course, but doing so can, I hope, offer some insight into the nature of the potential energy as well as into how the magnetic field is causing the fringe shift in the $\mathrm{AB}$ effect. The idea is that since these flux lines do not vanish in the presence of other flux lines, they will still be acting on the electron. Although one needs to generalize from magnetic and electric fields to electromagnetic, one can still suppose that lines of flux (4-lines of flux) are indestructible, and interact directly with charges at their locations.

Another intuitive picture by which one might motivate the view that the flux from individual charges can affect other charges comes this time from quantum field theory in its standard model of particle physics. One common explanation for the interaction of charges on the standard model is that the particles exchange the so-called force carriers - the gauge particles - of the theory. Without going too deeply into this, and with all due caveats about the proper interpretation of Feynman diagrams, and about the well-known interpretive problems of trying to give a particle ontology for quantum field theory, and even about the fact that the way in which we standardly calculate these forces is by passing to the potential formalism, one can see at least this: the action of these particles, in exchanging force carriers, takes place independently of the action of other particles in the system (assuming that the particles are not entangled). We treat the exchange between charges individually even for very complicated field configurations. Looked at this way, the action of the field on a moving charge is really the individual action of the many other charges in its vicinity. Exchange of a virtual photon (in the electrodynamical case) is the field action between just two 
charges, and it changes their phases accordingly.

Given these two intuitive stories then, we can see the potential as arising from the individual actions of the various components of the current, even in the case of no net external magnetic field. Again though, the facts of the matter seem to be independent of the persuasiveness or lack thereof that one finds in these stories. We can, whatever story one likes to tell, sum the individual terms arising from the individual current elements and even though the particle experiences no net force it still responds to the presence of the component charges of the current through its change in phase; the phase change itself arises from the time spent in the field, time spent with a given potential energy. Clearly we can do the same trick with the dual AB effect. There, again, there is no net force but we can see the potential energy as arising from a peculiar arrangement of charges, and the work required to produce that arrangement. Were we to consider the interaction between each charge in the Faraday cages and the electron wave components within those cages, we would find that the integration of the forces over the distance separating the various charges sums to $U$, the total potential energy.

We have now what I think is a clear proposal for how to eliminate all reference to potentials, and moreover a clear proposal for how to account for the phenomena in terms of well-established physical quantities - the magnetic and electric fields (electromagnetic field). We take the electromagnetic potential energy terms in the Lagrangian to express the electromagnetic field energy necessary to produce the field configuration, not the net field but rather the individual components of the field. Whatever one thinks of the intuition pumps above, it should be obvious that we can carry out our analysis in terms of electromagnetic fields alone - for the standard potential of a single charge is non-zero just in case its electromagnetic field is non-zero, and the potential energy is a Lorentz 
scalar in $\mathbf{E}$ and $\mathbf{B}$.

Let me pause here to make two clarifying points. First it may appear to be obvious that there is interaction between the solenoid and the external electron. For suppose that the net field inside the solenoid is constant, then there must be work done to keep it constant as the magnetic field of the electron increases at the location of the solenoid. Then suppose that the interior field is not constant, in that case the current will have to overcome an increasing back-reaction force. Suggestions of this sort that appeal to the possibility that the electron's magnetic field penetrates the solenoid are considered from time to time; see Boyer (2006) and references therein. Boyer also is challenging the orthodoxy that the $\mathrm{AB}$ effect cannot arise from local field interactions, but from a perspective significantly different from mine. If fact Boyer is attempting to account for the effect in terms of the scattering of the electron's magnetic field off the fields in the interior of the solenoid. There seems to be a consensus that Peshkin's critique of such programs is decisive (Peshkin and Tonomura, 1989). (Thanks to an anonymous referee for prompting me to highlight Peshkin's account.) However my proposal here is independent of the above issue, for I consider only the component magnetic fields exterior to the solenoid. However a related worry does arise concerning the possibility of magnetic fields exterior to the solenoid. In the most definitive tests of the effect by Tonomura et al. (Peshkin and Tonomura, 1989) the solenoid is enclosed in a superconducting shield. In that case the Meissner effect would prevent all magnetic fields from penetrating the superconductor and so there would be no magnetic fields at all in the path of the electron. (An anonymous referee has made this suggestion as did Yakir Aharonov in conversation.) But that is simply to assume the point at issue: whether there are component fields acting in a region in which the net fields vanish identically. The Meissner effect appears to be perfectly consistent with the vanishing of net but not component fields. For what but a redistribution of currents, and hence the fields that they produce, enforces 
the vanishing of the net field in the interior of the superconductor? Absent further argument, and I am not aware of any explicit treatment of this in the literature, there is simply no reason to think that the physics of superconductivity is at odds with the proposal that component fields are active in the exterior of the superconducting shield. If my proposal is correct then only a test along the lines of the $\mathrm{AB}$ effect would reveal the action of these component fields, and on my proposal that is just what the $\mathrm{AB}$ effect does reveal.

\subsection{The AB Lagrangian}

To show explicitly that it is possible to account for the AB effect in terms of the interaction between the individual charges, I now display that account for the simple case we have been considering. In our approximation of slowly changing and hence non-radiating fields, the appropriate Lagrangian is properly expressed in the Darwin form, which is correct to second order in $\frac{v}{c}$. This form is also instructive because it expresses the potential energy explicitly in terms of the interactions between the charges.

The Darwin Lagrangian for a charge, $a$, in the field produced by a collection of charges, for fixed motion of the other charges is:

$$
L_{a}=\frac{m_{a} v_{a}^{2}}{2}+\frac{1}{8} \frac{m_{a} v_{a}^{4}}{c^{2}}-e_{a} \sum_{b \neq a} \frac{e_{b}}{R_{a b}}+\frac{e_{a}}{2 c^{2}} \sum_{b \neq a} \frac{e_{b}}{R_{a b}}\left[\boldsymbol{v}_{a} \cdot \boldsymbol{v}_{b}+\left(\boldsymbol{v}_{a} \cdot \mathbf{n}_{a b}\right)\left(\boldsymbol{v}_{b} \cdot \mathbf{n}_{a b}\right)\right]
$$

Although messy, it is instructive to express the final term as an interaction between the moving charge, $a$ and the magnetic fields of the other charges. For a given charge $b$ : 


$$
\frac{e_{b}}{R_{a b}} \boldsymbol{v}_{b}=\left.\left.B_{b}\right|_{a} \frac{R_{a b} c \hat{\boldsymbol{v}}_{b}}{\sin \left(\theta_{R_{a b}, v_{b}}\right)} \stackrel{\text { def }}{=} B_{b}\right|_{a} \kappa
$$

Here $\left.B_{b}\right|_{a}$ is the magnetic field of the charge $b$ evaluated at the location of charge $a$. $\boldsymbol{\kappa}$ is a vector characterizing $b$ 's state of motion and location relative to $a$.

We may now express the Lagrangian as:

$$
L_{a}=\frac{m_{a} v_{a}^{2}}{2}+\frac{1}{8} \frac{m_{a} v_{a}^{4}}{c^{2}}-e_{a} \sum_{b \neq a} \frac{e_{b}}{R_{a b}}+\left.\frac{e_{a}}{2 c^{2}} \sum_{b \neq a} B_{b}\right|_{a} \kappa \cdot\left[\boldsymbol{v}_{a}+\mathbf{n}_{a b}\left(\boldsymbol{v}_{a} \cdot \mathbf{n}_{a b}\right)\right]
$$

Now we examine the terms on the right hand side of the equation. The first term is the kinetic energy of the moving charge to first order in $\frac{v}{c}$; the second term is the second order correction to the kinetic energy. The third term is the Coulomb interaction between the moving charge and each of the charges in the collection. Since the net charge vanishes, so too does this term in the Lagrangian. The last term is the magnetic interaction energy between the moving charge and the moving charges in the array.

In standard presentations of the AB effect (for example Quigg (1997) and indeed implicitly in Aharonov and Bohm's (1959)) it is recalled that if $\psi_{0}(t, \mathbf{x})$ is a given wave function of a free particle with charge $e$ in the absence of electromagnetic fields then in the presence of such fields:

$$
\psi(t, \mathbf{x})=e^{i S_{e m}} \psi_{0}(t, \mathbf{x})=e^{i \int^{t} L_{e m} d t^{\prime}} \psi_{0}(t, \mathbf{x})
$$


where $S_{e m}, L_{e m}$ are the action and Lagrangian respectively that arise from the interaction of the particle with the fields. ${ }^{6}$ We can then take the change in phase due to the electromagnetic interaction for a time, $t$, to be $\int_{0}^{t} L d t^{\prime}$ and the infinitesimal phase change to be $L d t$. And we can conclude that for otherwise free particles travelling different paths under the influence of electromagnetic fields that after a time $t$ the change in phase due to that influence is:

$$
\Delta \phi=\Delta \phi_{\psi 1}-\Delta \phi_{\psi 2}=\int_{0}^{t}\left(L_{e m 1}-L_{e m 2}\right) d t
$$

A suitable choice for the Lagrangian, and the one we normally choose is

$$
L_{e m}=\frac{e}{c} \mathbf{A} \cdot d \boldsymbol{v}-e \phi
$$

On the other hand, one is also free in this limit to make the choice of the Darwin Lagrangian. Therefore we can simply take

$$
\begin{aligned}
\psi(t, \mathbf{x})=e^{i \int_{0}^{t}-e_{a} \sum_{b \neq a} \frac{e_{b}}{R_{a b}}+\left.\frac{e_{a}}{2 c^{2}} \sum_{b \neq a} B_{b}\right|_{a} \boldsymbol{\kappa} \cdot\left[\boldsymbol{v}_{a}+\mathbf{n}_{a b}\left(\boldsymbol{v}_{a} \cdot \mathbf{n}_{a b}\right)\right] d t^{\prime}} & \psi_{0}(t, \mathbf{x}) \\
& =e^{i \int_{0}^{t} \frac{e a}{\left.2 c^{2} \sum_{b \neq a} B_{b}\right|_{a} \boldsymbol{\kappa} \cdot\left[\boldsymbol{v}_{a}+\mathbf{n}_{a b}\left(\boldsymbol{v}_{a} \cdot \mathbf{n}_{a b}\right)\right] d t^{\prime}} \psi_{0}(t, \mathbf{x})}
\end{aligned}
$$

for the situation described above.

\footnotetext{
${ }^{6}$ For an illuminating discussion of the action principle for electromagnetism see Landau and Lifshitz (1975) §§16, 26 , and 27.
} 
If we now assume, arguendo, that the phase of each of the components of the electron wave function is determinate at each instant ${ }^{7}$ we can see that the instantaneous change in the phase difference between the components is $\left(L_{\psi 1}-L_{\psi 2}\right) d t$. But note that $L_{\psi 1}$ and $L_{\psi 2}$ are, as we have shown, expressible in terms of the individual magnetic fields of the current elements. Thus it is possible to write the infinitesimal relative change in phase as a function of the magnetic interaction between the travelling electron and the charges in the solenoid. Then integrating over the time of travel will give the total phase difference between the components in agreement with the standard prediction for the $\mathrm{AB}$ effect. That is:

$$
\begin{aligned}
& \Delta \phi=\Delta \phi_{\psi 1}-\Delta \phi_{\psi 2}=\int_{0}^{t}\left(L_{e m 1}-L_{e m 2}\right) d t \\
&=\int_{0}^{t} \frac{e}{2 c^{2}}\left[\sum_{b \neq e_{1}} B_{b} \boldsymbol{\kappa} \cdot\left[\boldsymbol{v}_{e_{1}}+\mathbf{n}_{b e_{1}}\left(\boldsymbol{v}_{e_{1}} \cdot \mathbf{n}_{b e_{1}}\right)\right]-\sum_{b \neq e_{2}} B_{b} \boldsymbol{\kappa} \cdot\left[\boldsymbol{v}_{e_{2}}+\mathbf{n}_{b e_{2}}\left(\boldsymbol{v}_{e_{2}} \cdot \mathbf{n}_{b e_{2}}\right)\right]\right] d t^{\prime} \\
& \quad=\frac{e}{c} \int_{0}^{t}\left(\mathbf{A}_{1} \cdot \boldsymbol{v}_{1}-\mathbf{A}_{2} \cdot \boldsymbol{v}_{2}\right) d t^{\prime}=\frac{e}{c} \int_{0}^{x}\left(\mathbf{A}_{1} \cdot d \mathbf{x}_{1}-\mathbf{A}_{2} \cdot d \mathbf{x}_{2}\right)=\frac{e}{c} \oint \mathbf{A} \cdot d \mathbf{x}
\end{aligned}
$$

And this equation holds for any $\mathbf{A}$ whatever, since all differences between potentials vanish under loop integration.

Note that we have not "fixed a gauge" here. Gauge fixing is a mathematical operation that one carries out in choosing a particular vector potential consistent with Maxwell's equations and satisfying some desiderata or other. We have not done that. Instead we have written down an

\footnotetext{
${ }^{7}$ This is not strictly speaking necessary, though I think it is correct. We need only assume only that the instantaneous change in the relative phase between the beam components that is in fact due to the electromagnetic fields is itself determinate - that suffices to account for the effect itself in terms of the magnetic fields alone. The question of how to think about the phase remains. See Section 4 for a speculative and necessarily brief discussion of this point.
} 
expression characterizing the effect on the wave function that arises from what I am arguing are the actual magnetic fields that are present at the location of the electron. It is an interesting fact, worth some analysis, that one can translate this expression into one involving a particular choice of vector potential. But that fact is irrelevant to the present analysis. We have assumed at the outset some determinate wave function when the beam first divides, and we observe the change in phase due to the magnetic field that results from travel along the different paths. Of course one may choose to add terms to the Lagrangian of the form $\nabla \lambda$ or one may instead choose to add terms to the electron phase - and then these choices will have to be made mutually consistent. But one virtue of the preceding account is to make clear that such terms are merely additions allowed by the formalism that have nothing to do with the interactions between the charges.

Because of the way the terms in the Darwin Lagrangian arise they appear to correspond to terms in the non-relativistic Liénard-Wiechert potentials, which can be arrived at by imposing a Lorentz gauge condition. So one may well still wonder how this discussion does connect to various proposals for "fixing a gauge". The best way to look at the issue is, I think, to stop thinking of gauge as connected to electrodynamics itself and to think of it as part of our choice of representational strategy. There is an important mathematical relationship between how we represent the Lagrangian and how we represent the electron phase. There is, however, no obvious reason why one should connect that relationship directly to electrodynamics. Further discussion of this point is beyond my scope here. $^{8}$

The $\mathrm{AB}$ effect, and all of electrodynamics essentially, is encoded in the electromagnetic field strengths (together with the charge-current diistribution); for understanding the actual physics,

\footnotetext{
${ }^{8} \mathrm{I}$ am very grateful to an anonymous referee both for urging me to make the above discussion more explicit and to clarify the role of the Darwin Lagrangian in the application of the action principle, and also for helpful suggestions about how to do so.
} 
the potentials are an irrelevant artifact. The vector potential remains merely a powerful calculational device - as it was in the classical theory. At the same time note that the AB effect is a terrible device for measuring the actual current since it is sensitive only to the value of the holonomy and that under-determines the value of the current. It would seem an illegitimate return to verificationist scruples to assume that the only physics is the physics that is revealed directly by that effect.

We have now seen the AB effect accounted for entirely in terms of locally operative, deterministic, electrodynamical field strengths. I think the conclusion is obvious. We should not take seriously such quantities as the net field. When we do take these quantities seriously, and think that only the net field can act we are led naturally to think that in certain circumstances new fields are required. The fact that there is no net field however has very little to say about what processes are actually taking place. The construction laid out here shows clearly that there is a function, $f$, such that $\sum_{i} f\left(\mathbf{B}_{i}\right)=\Delta \phi$; whereas there is no $g$ such that $g\left(\sum_{i} \mathbf{B}_{i}\right)=\Delta \phi$. The sum of the effects cannot be rewritten as the effect of a sum. Because the peculiar geometry of the solenoid leads to the vanishing of the net magnetic field (and hence our normal measure of the field, the Lorentz force) we are tempted to think that there are no locally present magnetic fields. While the vanishing of the net field obscures the role of the magnetic field in changing the electron phase, the $\mathrm{AB}$ effect should remind us of just this fact: the phase of the wave function is affected not by net fields but by the individual field components. 


\subsection{Other gauge theories}

Before I conclude this section, I raise the question of how this story is to connect with our accounts of other gauge theories. First, the electromagnetic account - compelling physical story or nocertainly establishes my main claim: Gauge theories do not, in general, entail any fancy new metaphysics. But second I speculate that an exclusive focus on the mathematical form of the constraints arising in our physics is a mistake and that a more fruitful approach would involve explicitly addressing the origin of these constraints. We can learn much from the methods of gauge-theoretic analysis, but what we cannot learn directly through these methods is what the underlying fields are. When we focus on the gauge field itself as causally relevant we close off any route to an intrinsic causal account in terms of the underlying physical fields. The equations of motion follow from the constraints acting on the system, and the Lagrangian itself - but insight into the causally relevant formulation of the Lagrangian, and hence the causally relevant field does not follow. We have seen over the last several decades the fruitfulness of trying to extract deep insight into quantum electrodynamics from a consideration alone of its character as a gauge theory. Perhaps now it is time to turn our attention back to the underlying physical system itself - the currents and their electromagnetic fields. The general question of how to understand the application of Lagrangian methods to physical systems, though of crucial importance, is beyond the scope of this work.

\section{Interlude on the quantum phase}

A standard view of the role of the quantum phase is endemic in the literature, and a difficulty with this view for understanding the way physical systems are best to be represented in their interactions 
is clearly apparent in treatments of the $\mathrm{AB}$ effect. What follows is an attempt to overcome my own difficulty in coming fully to understand the quantum phase and is therefore quite tentative. All I have to offer is an analogy that may help make clear how I think the phase is best understood.

Consider the case of velocity. There is a sense in which relativity (Galilean and Einsteinian) has shown us that velocity is irreal. We are free, at each point on the trajectory of a particle, to choose the inertial frame in which we will describe the motion of that particle, and we are therefore free to assign to it any velocity we choose (but less than $c$, of course, in STR). In analogy with the phase case, I can adopt infinitesimal local inertial frames and knit them together in just the right way so that there are no observable consequences of my local choices. Doesn't this freedom then demonstrates that velocity is unreal? Not necessarily. Such freedom shows only that velocity is relative to a choice of inertial frame and, moreover, once I specify an inertial frame, no-one suggests that those forces in my frame of description that change the velocity of the particle in that frame are not really changing the velocity of the particle. It may be possible to make a time-dependent choice of frame such that the particle is always described as moving at the same velocity under that choice. But clearly the constancy of the particle's velocity is an artifact of my choice of representation scheme. The freedom I have to make this choice does not by itself suggest that there is something unreal about velocity.

Similarly the fact that I have complete liberty to specify locally the phase of a quantum system —as long as these local choices knit together properly where they overlap-should not suggest to me that the phase itself is unreal. The numerical value of the phase is certainly highly non-trivially influenced by my choices, but there is reason to think that there is a fact about the phase itself and the way it is being affected physically that is independent of these representational schemes. Two 
electron wave functions that arise from splitting a single wave function into two components have phases that are real, and that are similarly affected by the magnetic fields of the elements of the current in the solenoid. And as the time parameter increases, the phases have real significance for the behavior of the total system. So while the actual value of the phase at any point on the electron's path is not independent of my choice of phase assignment, the difference between the phase that arises from travelling distinct paths is determinate and independent of my phase convention.

That the phase of the electron components in the $\mathrm{AB}$ effect are only specified relative to each other no more suggests that their phases are unreal than does the relativity of velocity suggest that velocity itself is unreal. It is then, I maintain, perfectly appropriate to treat the change in phase along the electron path as determinate at each point on that path. This treatment involves reifying the phase, but not making distinct wave functions out of wave functions differing only in phase value (as Leeds (1999) for example fears). Nothing in my account should suggest that I take the value of the phase to be fixed by the theory. One is still free to assign a phase transformation, but one needn't think of that as having anything to do with electrodynamics itself. Rather such a transformation requires its own complementary, compensatory term in the Lagrangian.

\section{Conclusion}

One might not be persuaded by the physical explanation of the Lagrangian term above. Indeed, an obvious objection to the above story is the following: Why should we think of the magnetic field as changing the phase, and more generally, why should the Lagrangian be modified by that factor

rather than by the potential term as is more traditional? Even granting that we can calculate the lines of flux as claimed, why should those be part of our causal story? 
To this objection there is little to respond that is truly decisive. But the following considerations are relevant, and I think powerful. First, why should the vector potential itself modify the Lagrangian? Is it only because it arises in the mathematical form that it does - as that quantity vector calculus tells us must arise whenever there is a divergenceless field in the neighborhood, or a quantity that has the right transformation properties? But if that is our only motivation for using the potential term, then it has no conceptual advantage over the more familiar electromagnetic field. If that is right, then having a conceptual story about the action of the fields of the individual elements of current should be an advantage. That advantage is enhanced when we note that the story in terms of magnetic field components is completely well-defined, gauge independent, and local. (And for those for whom such is itself an advantage, the account is ontologically parsimonious.)

There is a kind of blend of prospective and retrospective physical legitimacy account to be given here. We do, in fact, as the vast literature on the AB effect makes clear, consider the electromagnetic field strengths to be better physical quantities than the potentials. All things being equal, a story told in terms of electromagnetic fields should be more compelling than one that relies on the unphysical potential terms. Given the mathematical form of the account above, the insight that the density of flux experienced by the electron is at the root of its phase shift arises naturally.

I merely highlight now the following claim, but I will not here argue for it in detail. The story as I have told it in terms of current elements is not restricted to any particular account of the microphysics. As long as the smallest portions of any bulk matter that gives rise to magnetic fields are treated as themselves giving rise to sub-components of the net field, the technique above will work as advertised. For the traditional potentials can themselves be generated from the infinitesimal charge-current elements, and these infinitesimal components can be re-conceived as generating the 
infinitesimal components of the flux field. And all of the standard story underlying the AB effect does treat the bits of matter in bulk as required on such a view.

A serious question remains however. How is it that the fields arising from the individual elements do not simply vanish? How can they continue to be causally active when their net field vanishes? Here another analogy might help. When we illustrate the force composition law for mechanical systems, it is sometimes useful to consider many strings attached to a single mass. As forces are applied to these various strings and equilibrium is reached, we do not claim that there is no force acting on the mass. Instead, we calculate the acceleration of the mass by finding the result due to each individual string. The strings are still there, as are the components of the force. Even more relevant is the case of a boat tied at dock. If the tensions in the various ropes are such that their vector sum vanishes, then the boat will be stationary. But it would be madness to think that no forces are acting on the boat. Clearly sufficient tension in the ropes will tear the boat apart. (Similarly, as a colleague has quipped, the assurance that no net forces are present will be cold comfort to a prisoner sentenced to be drawn and quartered - our victim is torn apart in any case.)

I claim that something relevantly analogous is happening in the case of electrodynamics; I claim that the individual field elements are still present and that they interact individually with the electron as though it were in the flux field whose action is as I have described above. Naturally the electron is not torn apart, but its phase does change - and continues to change even when no net force is acting.

Consider how one might react who is ignorant of the history of this debate: When told about the Lagrangian for electromagnetism she might reply that the $\mathbf{A}$ term appearing there is simply a mathematical convenience, but that what is really going on arises from the $\mathbf{B}$ field. Challenged, 
she might follow just the line of argument outlined above and claim that something to do with the magnetic flux density along the path of a charge is the relevant term to add to the Lagrangian. One important lesson to be drawn from this discussion is that it is sensible to suppose that the net field is fictional while the component field is factual. But how can this be? First note that there is nothing the net field does that the components of the field together cannot do - if there is a net force it is equal in strength to the vector sum of the component forces; the radiative propagation of the net magnetic field is equal in magnitude to the sum of the radiation from the components fields; etc. But here we have found something else that can be done by the component fields that manifestly cannot be done by the net field.

So since we know that the individual bits of charge-current are still producing their own fields (how else could a net field arise?) why should we hesitate to recognize the distinction between net and component fields, and accord significance only to the latter? On the other hand in light of the well-known worries over non-locality, etc., that arise out of standard accounts of the effect, it is perhaps no longer sensible to suppose the contrary and to insist that, since the net field cannot produce all of the relevant physical effects, there must be some novel entity that does. At any rate, there is no particular pressure on us to adopt new metaphysics in the light of the Aharonov-Bohm effect. Surely this is what Faraday would reply. 


\section{References}

Aharonov, Y. and Bohm, D. (1959). Significance of electromagnetic potentials in the quantum theory. Physical Review, 115 (3): 485-491

Aharonov, Y. and Bohm, D. (1962). Remarks on the possibility of quantum electrodynamics without potentials. Physical Review, 125 (6), 2192-2193.

Belinfante, F. (1962). Consequences of the postulate of a complete commuting set of observables in quantum electrodynamics. Physical Review, 128 (6), 2832-2837.

Boyer, T. ( 2006). Darwin-Lagrangian analysis for the interaction of a point charge and a magnet: considerations related to the controversy regarding the AharonovBohm and AharonovCasher phase shifts. Journal of Physics A, 39, 3455-3477.

Belot, G. (1998). Understanding electromagnetism. British Journal for Philosophy of Science, 49 (4), 531-555.

Cao, T. (1999) (ed.). Conceptual foundations of quantum field theory (pp. 298-309). Cambridge: Cambridge University Press.

DeWitt, B. (1962). Quantum theory without electromagnetic potentials. Physical Review 125 (6), 2189-2191.

Healey, R. (1997). Non-locality and the Aharonov-Bohm effect. Philosophy of Science, 64, 18-41.

Healey, R. (1999). Is the Aharonov-Bohm effect local? In Cao, T. (1999).

Healey, R. (In press). Gauging what's real. Cambridge: Cambridge University Press.

Jackson, J. (1975). Classical electrodynamics. New York: John Wiley \& Sons.

Landau, L. and Lifshitz, E. (1975). The classical theory of fields. New York, NY: Elsevier.

Leeds, S. (1999). Gauges, Aharonov, Bohm, Yang, Healey. Philosophy of Science (4), 66, 606-627.

Martin, C. (2002) Gauge principles, gauge arguments, and the logic of nature", Philosophy of Science, 69 (3), S221-S234.

Mattingly, J. (2006). Which gauge matters. Studies in History and Philosophy of Modern Physics, $37,243-262$.

Peshkin, M. and Tonomura, A. (1989). The Aharonov-Bohm effect, lecture notes in physics 340. New York, NY: Springer-Verlag.

Quigg, C. (1997). Gauge theories of the strong, weak, and electromagnetic interactions. Reading, 
MA: Addison-Wesley. 